\title{
Acronyms Used in the Text
}

ACDIP Asociación Campesina del Departamento del Petén

ACROX

ACSUR

ADISC

ADRI

AMR

ANC

ANN

ASC

ASOCODE

AVANCSO
Petén Campesino Association

Asociación Campesina Rosario Xolhuitz

Rosario Xolhuitz Campesino Association

Asociación para la Cooperación con el Sur

Association for Cooperation with the South

Asociación de Desarrollo Integral Santa Cruz

Santa Cruz Association for Comprehensive

Development

Alianza de Desarrollo Rural Integral

Alliance for Comprehensive Rural Development

Alianza de Mujeres Rurales

Alliance of Rural Women

Asociación Nacional Campesina

National Campesino Association

Alianza Nueva Nación / Alternativa Nueva Nación

Alliance for a New Nation / Alternative for a

New Nation

Asamblea de Sociedad Civil

Civil Society Assembly

Asociación Centroamericana de Organizaciones

Campesinas para la Cooperación y el Desarrollo

Central American Association of Campesino

Organizations for Cooperation and Development

Asociación para el Avance de las Ciencias Sociales en

Guatemala

Association for the Advancement of the Social

Sciences in Guatemala 
xiv Acronyms Used in the Text

CACIF

CAFTA-DR

CCDA

CEH

CDN

CICIG

CLOC

CNAIC-P

CNCG

CNOC

CNP-T

CNUS

COCODE

CODECA

CONAMPRO
Comité Coordinador de Asociaciones Agrícolas, Comerciales, Industriales, y Financieras

Coordinating Committee of Agricultural, Commercial, Industrial, and Financial

Associations

Dominican Republic-Central America Free Trade

Agreement

Comité Campesino del Altiplano

Campesino Committee of the Highlands

Comisión para el Esclarecimiento Histórico

Historical Clarification Commission

Consejo de Dirección Nacional

National Directive Council

Comisión Internacional Contra la Impunidad en

Guatemala

International Commission against Impunity in

Guatemala

Coordinadora Latinoamericana de Organizaciones del

Campo

Latin American Coordinator of Rural

Organizations

Consejo Nacional Indígena-Campesina y Popular

National Indigenous-Campesino and Popular

Council

Confederación Nacional Campesina de Guatemala

National Campesino Confederation of Guatemala

Coordinadora Nacional de Organizaciones Campesinas

National Coordinator of Campesino Organizations

Coordinación Nacional Permanente sobre Derechos

Relativos a la Tierra de los Pueblos Indígenas

Permanent National Coordinator on Rights

Related to Land and Indigenous Peoples

Comité Nacional de Unidad Sindical

National Committee on Labour Union Unity

Consejo Comunitario de Desarrollo

Community Development Council

Comité de Desarrollo Campesino

Campesino Development Committee

Coordinadora Nacional de Pequeños y Medianos

Productores 
National Coordinator of Small and Medium

Producers

CONAP

Consejo Nacional de Áreas Protegidas

National Council for Protected Areas

CONATIERRA Comisión Nacional de Tierras

National Land Commission

CONDEG

Comité Nacional de los Desplazados de Guatemala

Guatemalan National Committee of the Displaced

CONGCOOP Coordinación de ONG y Cooperativas

Coordinator of NGOs and Cooperatives

CONIC

CONTIERRA Dependencia Presidencial de Asistencia Legal y

Coordinadora Nacional Indigena y Campesina

National Indigenous and Campesino Coordinator

Resolución de Conflictos sobre la Tierra

Presidential Office for Land Conflict Legal

Assistance and Resolution

CRD Convergencia para la Revolución Democrática

Convergence for the Democratic Revolution

CTG Confederación de Trabajadores de Guatemala

Confederation of Guatemalan Workers

CUC Comité de Unidad Campesina

Committee for Campesino Unity

DED Deutscher Entwicklungsdienst

German Development Service

EGP Ejército Guerrillero de los Pobres

Guerrilla Army of the Poor

FAR Fuerzas Armadas Rebeldes

Rebel Armed Forces

FDYEP Empresa de Fomento y Desarrollo del Petén

Petén Promotion and Development Agency

FEDECOCAGUA Federación de Cooperativas Cafetaleras de Guatemala

Guatemalan Federation of Coffee Cooperatives

FLACSO Facultad Latinoamericana de Ciencias Sociales

Latin American Faculty of Social Sciences

FNL Frente Nacional de Lucha

National Struggle Front

FOGUAVI Fondo Guatemalteco para la Vivienda

Guatemalan Housing Fund

FONAPAZ Fondo Nacional para la Paz

National Peace Fund 
xvi Acronyms Used in the Text

FONTIERRAS Fondo de Tierras

Land Trust Fund

FUNDAECO Fundación para el Ecodesarrollo y la Conservación

Foundation for Ecodevelopment and

Conservation

IBRD International Bank for Reconstruction and

Development

INE Instituto Nacional de Estadística

National Statistics Institute

INTA Instituto Nacional de Transformación Agraria

National Institution for Agrarian Transformation

MAGA

Ministerio de Agricultura, Ganadería, y Alimentación

MLAR

Ministry of Agriculture, Cattle, and Food

MSICG

MST

Market-led agrarian reform

Movimiento Sindical, Indígena y Campesina

Guatemalteco

Guatemalan Labour, Indigenous, and Campesino

Movement

Movimento Dos Trabalhadores Rurais Sem Terra

Landless Workers' Movement

ORPA Organización Revolucionaria del Pueblo en Armas

Revolutionary Organization of the People in Arms

PTI Pastoral de la Tierra Interdiocesana

Interdiocesan Land Pastoral

PTSM Pastoral de la Tierra Interdiocesana de San Marcos

Interdiocesan Land Pastoral of San Marcos

RIC Registro de Información Catastral

Cadastral Information Registry

SAA Secretaría de Asuntos Agrarios

Secretariat of Agrarian Affairs

UASP Unidad de Acción Sindical y Popular

Union for Labour and Popular Action

UNAGRO Unión Nacional de Agricultores

National Farmers Union

URNG Unidad Revolucionaria Nacional Guatemalteca

Guatemalan National Revolutionary Unity

USAID United States Agency for International

Development

UVOC Unión Verapacense de Organizaciones Campesinas

Verapaz Union of Campesino Organizations 